\title{
The Comeback of A Rare Liver Disease-Indian Childhood Cirrhosis:
} A Case Report

\author{
Authors \\ Dr R. Sindhu', Dr Fairy Susan Varghese ${ }^{2}$, Dr Sunil Kumar Agarwalla ${ }^{3}$ \\ Dr S.K. Behera ${ }^{4}$ \\ ${ }^{1}$ M.B.B.S, Post graduate in Department of Pathology. \\ ${ }^{2}$ M.B.B.S, Post Graduate in Paediatrics \\ ${ }^{3}$ M.D, Associate Professor in Paediatrics \\ ${ }^{4}$ M.D, Assistant Professor in Pathology
}

Dept of Pathology and Dept of Paediatrics, MKCG Medical College, Berhampur, Odisha 760004, INDIA Corresponding Author

Dr R. Sindhu

Department of Pathology, M.K.C.G Medical College, Berhampur, Odisha 760004

Email: sindhushankar51@gmail.com,Ph: 7854831852

\begin{abstract}
Indian Childhood Cirrhosis (ICC) is a chronic childhood liver disease due to the deposition of copper in the liver. It primarily affects children of one to three year age group. Predisposing factors include genetic factors, infections and copper intoxication due to cooking food in copper containing utensils. The disease which had a high case fatality in the past became preventable, treatable and rare over the years. Recently a significant decline in the incidence of ICC has been observed in all parts of India due to reduction in the use of copper and brass utensils for boiling milk. Here we report a case of ICC which carries ample importance since it marks the comeback of an entity which was once considered to have been wiped out from the country.

Keywords: Indian childhood cirrhosis, liver disease, copper intoxication.
\end{abstract}

\section{Introduction}

ICC is a rare chronic liver disease confined to the Indian subcontinent. It has been described as 'a disease which grows worse in spite of all treatment eventually terminating in the death of the patient'. Though it was a common disease with high fatality rate in the past, over the last three decades the disease prevalence has drastically gone down. Unfortunately there are few cases emerging presently and this case represents one of them.

\section{Case Report}

An 18 month old male child, the first order product of a non consanguineous marriage was brought to the outpatient department with chief complaints of blood in stool since three days, vomiting of blood and fever of two days. Past history revealed that at the age of 13 months he had jaundice for which medications were taken from local hospitals with poor outcome.

The child had a normal vaginal hospital delivery, with antenatal, natal and postnatal events being 
uneventful. The child was not on any regular medications, and family history was insignificant. On examination, the child was moderately built and nourished with a protuberant abdomen. Gastrointestinal tract examination revealed a distended abdomen with liver enlarged five $\mathrm{cm}$ below the right costal margin (firm in consistency with sharp leafy margins).Rest of the systemic examination was normal. Ophthalmic examination did not reveal cataract or Kayser Fleischer rings.

Lab investigations showed: CBC: microcytic hypochromic anemia with acute infection, LFTSGOT-270 IU/l, SGPT- 256 IU/l, Serum Alkaline phosphatase-989.3 IU/l. Ultrasonography of the abdomen and pelvis showed moderate ascites and hepatomegaly with coarse echotexture of the liver. A trucutliver biopsy was done. The liver biopsy (H\&E stain) revealed degeneration of hepatocytes with variable amount of fibrosis and formation of regenerative nodules (micro nodular cirrhosis) (Fig 1). Rubeanic acid stain showed accumulation of intracytoplasmic copper in the hepatocytes thus confirming the diagnosis of Indian childhood cirrhosis (ICC) (Fig 2). The poor prognosis of the condition was explained to the parents and the child was referred to a higher centre with suggestions for liver transplantation as the only option for complete cure.

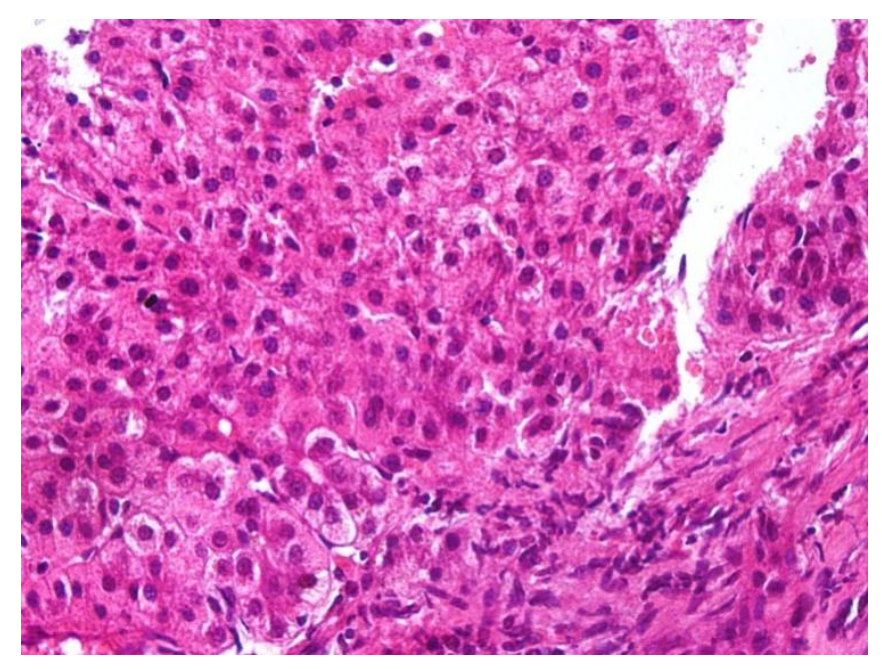

Figure 1: Microsection showing regenerative nodules of hepatocytes with fibrosis (H\&E X 400x)

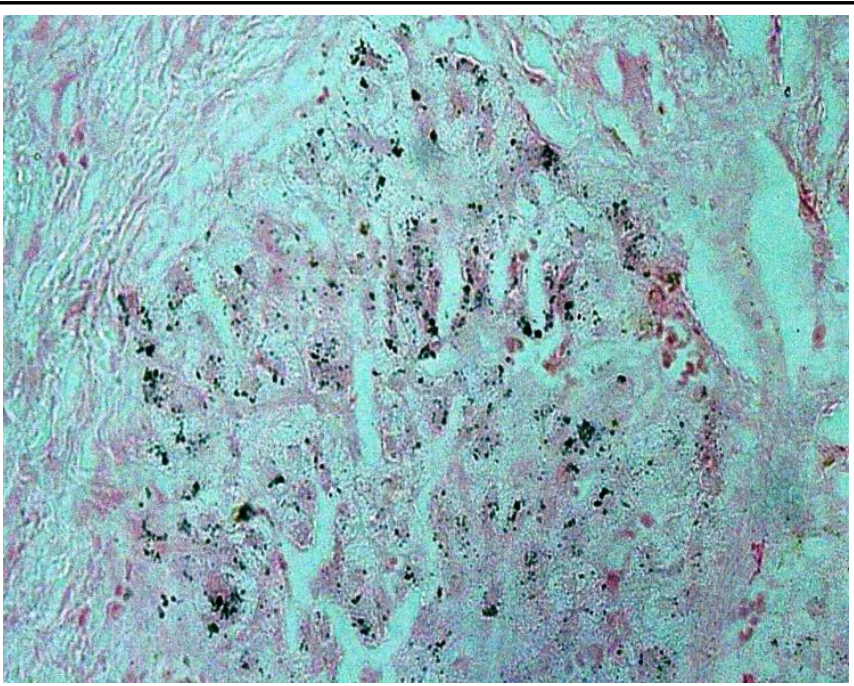

Figure 2: Intracytoplasmic copper accumulation in hepatocytes (Rubeanic acid stain X400x)

\section{Discussion}

Of the cirrhosis that affect Indian children, ICC is a discrete clinical and histologic entity in which large amounts of copper gets deposited in the liver. This unusual serious disorder was first reported from Calcutta in 1880's. ${ }^{1}$ During early 1930 's the disease was called as 'Infantile cirrhosis' or 'infantile biliary cirrhosis'.

The cause of the increased hepatic copper concentration in newborn is poorly understood. Since the newborn has a modestly increased hepatic copper concentration, and an impaired ability to excrete a copper it is possible that the newborn may exhibit increased hepatic copper storage and hepatotoxicity. An inherited predisposition to ICC has been suspected because of random occurrence of disease among siblings and death due to liver disease in second line family members. But there is no clear pattern of inheritance due to poor penetrance of disease. Also, mutation of gene associated with copper metabolism has not yet been identified.

Usually, ICC affects between ages nine months and fiveyears. ${ }^{3}$ the liver has a characteristic hard consistency and sharp edge. Jaundice is often the symptom precipitating admission, and usually heralds the terminal stage of disease. 
Clinically, ICC cases are classified into three categories: ${ }^{4,5}$

1. Highly suspected ICC - Hard liver along with any three from among clinical manifestation of hepatocellular dysfunction, portal hypertension, and sibling/family member death due to liver disease;

2. Suspected ICC - Firm liver with palpable spleen, distension of abdomen, ascites and/or oedema, sibling history present or not;

3. Early ICC - Firm liver with sibling/family history of liver disease and symptoms of poor appetite, sticky stools, and bowel movement abnormality.

Since, there are quite a lot of causes for hepatomegaly and cirrhosis in childhood, liver biopsy remains diagnostic and the only mode for definitive diagnosis of ICC.

The Indian Council of Medical Research (ICMR) had classified ICC based on the histological characteristics as such: ${ }^{6}$

1. Definitive ICC $($ DICC $)=$ Established, advanced disease showing moderate to marked hepatocyte damage with Mallory hyaline, interstitial fibrosis with or without micro nodules and moderate to marked copper deposit;

2. Probable ICC $(\mathrm{PICC})=$ Early, mild disease showing mild to moderate hepatocyte damage, no Mallory hyaline, some interstitial and septal fibrosis, and copper in less than $25 \%$ cases, mostly mild deposit;

3. Inactive cirrhosis $(\mathrm{CIRR})=$ Quiescent disease showing minimal or mild hepatocyte damage, cirrhosis mostly with micro nodules, no Mallory hyaline and copper in less than $10 \%$ cases in small amounts;

4. Abnormal Liver Unclassified (ALU) = Mild non-specific changes;

5. Normal= No obvious abnormality seen
Another condition where copper gets accumulated in hepatocytes is Wilson's disease. Interestingly, while the amount of hepatic copper in ICC was found to be far in excess of that seen in Wilson's disease, the former disease lacked most clinic pathologic features of the latter. Serum ceruloplasmin levels remain normal in cases of ICC unlike in Wilson's. ICC mostly affects infants and young children while Wilson's disease almost never occurs below four year of age and unlike the latter disease ICC is quickly fatal. ${ }^{7}$ Also, in Wilson's disease hepatocytic injury is neither not that severe nor has so much Mallory hyaline as in ICC which characteristically lacks the steatosis in livers of alcohol induced and Wilson's disease.

The common complications include appearance of jaundice, ascites, and chest infection which ends in death of the child with a mortality rate of $45 \%$ within 4 weeks. ${ }^{(8)}$ Penicillamineis found to be useful in early disease. But, adverse prognostic factors, particularly jaundice, should be recognized as indication for early liver transplantation. ${ }^{8}$

\section{Conclusion}

Thus in so-called 'cryptogenic' liver disease in a child, a diagnosisof ICC must be considered and liver biopsy must be done to evaluate the histologic abnormalities. Such overall scrutiny is needed to provide information on incidence and prevalence of the disease and to unveil some of the new facets of the disease regarding its etiopathogenesis and mode of inheritance.

\section{References}

1. Sen B. Infantile cirrhosis. Indian Med Gaz1887;22:338-42.

2. Achar ST, Raju VB, Sriramachari S. Indian childhood cirrhosis. J Pediatr1960;57:744-58.

3. Portmann B, Tanner M S, Mowat A P, Williams R. Orcein-positive liver deposits in Indian childhood cirrhosis. Lancet 1978;i:1338-40. 
4. Parekh SR, Patel BD. Epidemiologic survey of Indian childhood cirrhosis. Indian Pediatr1972;9:431-9.

5. Patel BD, Parekh SR, Chitale AR. Histopathological evolution of Indian childhood cirrhosis with emphasis on criteria of early diagnosis. Indian Pediatr1974;11:19-28.

6. Indian Childhood Cirrhosis (ICC) - A Multicentre National Collaborative Study. S. Sriramachari, editor. New Delhi: Indian Council of Medical Research; 2006.

7. N.C. Nayak\& A.R. Chitale. Indian childhood cirrhosis (ICC) \& ICC-like diseases:The changing scenario of facts versus notions.Indian J Med Res 2013(6);137:1029-42

8. M Stuart Tanner. Role of copper in Indian childhood cirrhosis. Am J ClinNutr 1998;67:1074S-81S. 